\title{
Journal of

\section{Towards commercial products by nanocasting: characterization and lithium insertion properties of carbons with a macroporous, interconnected pore structure ${ }^{\dagger}$}

\author{
Birte Jache, ${ }^{a}$ Christian Neumann, ${ }^{b}$ Jörg Becker, ${ }^{b}$ Bernd M. Smarsly ${ }^{a}$ and Philipp Adelhelm*a \\ Received 9th February 2012, Accepted 27th March 2012 \\ DOI: $10.1039 / c 2 j m 30787$ a
}

\begin{abstract}
Carbon materials with defined porosity are prepared using the nanocasting approach. The structural properties of the prepared carbon materials are examined by SEM, XRD, XPS, elemental analysis, nitrogen physisorption and $\mathrm{Hg}$ porosimetry. The materials exhibit an interconnected porous network with spherical pores in the macropore range, being a replica of spherical $\mathrm{SiO}_{2}$ particles. The average macropore size $(300-700 \mathrm{~nm})$ and surface area $\left(35-470 \mathrm{~m}^{2} \mathrm{~g}^{-1}\right)$ can be tailored by the choice of template and carbon precursor. More importantly, based on silica templates prepared by flame pyrolysis, the whole process, including HF etching of the template, can be easily industrialized. Lithium storage measurements are used to demonstrate the beneficial transport properties of the porous carbon materials which are referenced against non-porous carbons. The porous carbon materials exhibit high capacity (550 $\mathrm{mA} \mathrm{h} \mathrm{g}^{-1}$ at $\mathrm{C} / 5$ ) and excellent rate capability ( $90 \mathrm{~mA} \mathrm{~h} \mathrm{~g}^{-1}$ at $\left.60 \mathrm{C}\right)$. Surprisingly, the excellent lithium storage properties are related to the macroporous framework rather than high surface area and/or micro- and mesoporosity.
\end{abstract}

\section{Introduction}

The wide use of carbon materials in industrial and special applications is usually related to the fact that their microstructure and porosity can be tailored over a wide range.

A special class of carbon materials is accessible by using templating strategies. Here, porosity in the desired range is achieved by using sacrificial templates. A well known procedure is the so-called nanocasting process enabling the preparation of carbon materials with defined porosity - especially in the mesopore range - that is otherwise difficult to realize. In the hard templating approach, a carbon precursor is carbonized in the presence of a sacrificial template, usually $\mathrm{SiO}_{2}$, which subsequently is removed by chemical etching to yield a porous carbon material. ${ }^{1,2}$ Depending on the nature of the template (e.g. its amount, pore size or geometry) the porosity of the resulting carbon material can be adjusted over a wide range. Using this approach, plenty of carbon materials with defined pore size and geometry have been prepared that serve as excellent reference systems to study size effects and the influence of porosity on the materials performance. Carbon materials with ordered

${ }^{a}$ Institute of Physical Chemistry, Justus-Liebig-University Giessen, Heinrich-Buff-Ring 58, 35392 Giessen, Germany. E-mail: philipp. adelhelm@uni-giessen.de

${ }^{b}$ Heraeus Quarzglas GmbH \& Co. KG, Reinhard-Heraeus-Ring 29, 63801 Kleinostheim, Germany

$\dagger$ Electronic supplementary information (ESI) available. See DOI: $10.1039 / \mathrm{c} 2 \mathrm{jm} 30787 \mathrm{a}$ mesopores of a few nanometers in size, e.g. replicas of SBA-15 type silica, show improved properties as active material and support in battery and supercapacitor applications, hydrogen storage and catalysis, for example. ${ }^{3-7}$ The progress in the field of carbon materials with tailored porosity is also well documented in the literature. ${ }^{8-12}$ Another important factor governing the final properties of carbon materials is the type of carbon precursor used. Frequently used precursors such as sucrose or furfuryl alcohol lead to a more disordered carbon microstructure which intrinsically causes microporosity. Pitches carbonize comparatively well and a carbon microstructure with fewer defects is achieved featuring pronounced chemical stability and higher conductivity. ${ }^{13}$ Depending on the application in mind, one or the other precursor can be more advantageous. Concerning anode materials for battery applications we recently found that a hierarchical, interconnected structure with pores in the macro- and mesopore range and mesophase pitch as a carbon precursor is beneficial in achieving high capacity and excellent rate capability. ${ }^{14-16}$ Similar results are also found for other template based carbon structures with high surface area and well defined morphology. ${ }^{7,17}$

Even though many promising results have been reported for carbon materials prepared by the hard templating process, to the best of our knowledge no commercial product has been developed so far. This is because a cost-effective industrial scale-up is generally perceived to be difficult as the process involves a multistep procedure including infiltration of the template, carbonization and HF etching. To tackle this issue, we prepared porous 
carbon materials based on hard templating of fused spherical silica particles. The monolithic silica template offers the preparation of porous carbon replicas with tailored porosity at relatively low cost. Based on this template and the nanocasting process, Heraeus Quarzglas recently filed a patent application to prepare a special porous carbon to be commercialized under the registered trademark Porocarb ${ }^{\circledR} .{ }^{18}$ The main advantages of the process are: (1) the silica particle size can be adjusted, i.e. the carbon pore size can be tailored, and (2) the silica particles are fused, i.e. pore linkage within the carbon matrix is assured. The process can be scaled up and batches on the kilogram scale have already been produced at this early stage of fabrication. The carbon material is especially designed for applications where defined and interconnected porosity is needed.

In this paper, we first present the structural characterization of the silica templates and their carbon replicas. Secondly, we demonstrate the use of the carbon materials as anode material in lithium-ion batteries and comment on the influence of porosity and the carbon precursor used.

\section{Experimental section}

\subsection{Sample preparation}

The porous carbon samples were prepared by nanocasting using an approach earlier reported. ${ }^{13,14}$ Mesophase pitch (MP, Mitsubishi Gas Chemical Company) was dissolved in THF (technical grade) using an orbital shaker. The final solution was obtained by centrifugation (4000 rpm, $5 \mathrm{~min}$ ) followed by decantation. The MP content of the supernatant was approx. $10 \mathrm{wt} \%$. Silica samples (Heraeus Quarzglas GmbH \& Co. KG) were infiltrated by the MP solution in open polystyrene vessels under mild agitation while evaporating the solvent. After drying at room temperature, the silica-MP composites were carbonized at $\vartheta=$ $700{ }^{\circ} \mathrm{C}$ under $\mathrm{N}_{2}$ atmosphere ( $6 \mathrm{~h}$ ramp, $6 \mathrm{~h}$ dwell time). The porous carbon materials were obtained by dissolution of the silica template in hydrofluoric acid. CarbonSUC was provided by Heraeus Quarzglas GmbH. Graphite (SFG-44) was provided by Timcal Ltd.

\subsection{Electrode preparation and electrochemical measurements}

Carbon samples were ground to powder in a vibratory micro-mill (Pulverisette 0, Fritsch). Electrode slurries were made from carbon, polyvinylidene fluoride (PVDF, Solef 1310) and $N$-methyl-2-pyrrolidone (NMP). The contents of carbon and binder were $90 \mathrm{wt}^{\%} \%$ and $10 \mathrm{wt} \%$, respectively. Electrodes were prepared by doctor blading the slurry onto copper foil $(d=$ $10 \mu \mathrm{m})$. The thicknesses of the electrodes were approximately $40 \mu \mathrm{m}$. Circular electrodes $(d=1.2 \mathrm{~cm})$ were punched out and contained approx. $3 \mathrm{mg}$ of active mass each. Before transferring the electrodes to the glove box, they were further dried at $\vartheta=$ $120{ }^{\circ} \mathrm{C}$ for $2.5 \mathrm{~h}$ under vacuum to evaporate residual NMP and adsorbed water. Three-electrode Swagelok type cells were assembled in a glove box (MBraun Labmaster sp) under Ar atmosphere. Metallic lithium was used as counter- and referenceelectrode. Whatman glass microfiber filters ( $\mathrm{GF} / \mathrm{A})$ were used as separators. A $1 \mathrm{M}$ solution of $\mathrm{LiPF}_{6}$ in $1: 1 \mathrm{w} / \mathrm{w}$ EC:DMC (LP30, SelectiLyte, Merck) was used as electrolyte. Electrochemical measurements were conducted at room temperature using a Maccor (Model 4300) battery cycler. Cells were cycled galvanostatically (constant current, CC) at different C-rates between $0.01 \mathrm{~V}$ and $2.00 \mathrm{~V}$. The C-rate was calculated based on the theoretical capacity of graphite $\left(Q=372 \mathrm{~mA} \mathrm{~h} \mathrm{~g}^{-1}\right)$, i.e. $1 \mathrm{C}$ corresponds to a current of $i_{\mathrm{CC}}=372 \mathrm{~mA} \mathrm{~g}(\mathrm{C})^{-1}$. A potentiostatic step (constant voltage, $\mathrm{CV}$ ) was added after galvanostatic Li-insertion at $0.01 \mathrm{~V}$ until the current $i_{\mathrm{CV}}$ reached $i_{\mathrm{CV}}=i_{\mathrm{CC}} / 2$. Total Li-insertion capacities including the potentiostatic step are given as $Q_{\text {total }}$; Li-insertion capacities originating from the galvanostatic step are given as $Q_{\mathrm{CC}}$. Capacities are given in $\mathrm{mA} \mathrm{h}$ per gram of carbon ( $\left.\mathrm{mA} \mathrm{h} \mathrm{g}(\mathrm{C})^{-1}\right)$.

\subsection{Characterization methods}

SEM images were taken with a Zeiss LEO 982 FEG scanning electron microscope. A VarioMICRO cube (Elementar Analysensysteme $\mathrm{GmbH}$ ) was used for CHNSO elemental analysis. Nitrogen physisorption measurements were conducted at $T=$ $77 \mathrm{~K}$ using a Quantachrome instruments Autosorb 1. BJH pore size distributions were calculated from the adsorption branch of the isotherm. Samples were pre-dried overnight under vacuum at $\vartheta=120^{\circ} \mathrm{C}$. ThermoFisher scientific Pascal 140 and Pascal 440 machines were used for the mercury porosimetry measurements. X-ray photoelectron survey spectra were taken on a PHI 5000 Versa Probe (Physical Electronics). WAXS patterns were recorded on a Siemens D500 powder diffractometer in an angular range between $10^{\circ}<2 \theta<80^{\circ}$.

\section{Results and discussion}

\subsection{Concept}

The concept of the hard templating synthesis for the specific silica template and carbon precursor is sketched in Fig. 1. The silica template consists of spherical, fused $\mathrm{SiO}_{2}$ primary particles that form a porous framework in monolithic shape which in turn is synthesized by a flame pyrolysis process where a silica precursor ( $\mathrm{SiCl}_{4}$, for example), $\mathrm{H}_{2}$ and $\mathrm{H}_{2} \mathrm{O}$ are introduced into a hot flame (Fig. S1†). ${ }^{19-21}$ During combustion, $\mathrm{SiO}_{2}$ proto-particles develop which aggregate into larger clusters within the propagation of the turbulent flame. Sinter necks develop between single particles to form aggregates. These aggregates are collected on a rotating substrate where additional sinter necks develop, finally forming porous layers with defined thickness and porosity. The properties of the porous layers can be controlled by silicon precursor feed rate, flame temperature and other deposition parameters.

In the present process, the silica bodies are infiltrated by a solution $(\sim 10 \mathrm{wt} \%)$ of mesophase pitch (MP) dissolved in tetrahydrofuran (THF). After infiltration of the porous framework, the solvent is removed by evaporation under mild agitation. Consequently, the pitch covers the surface of the silica particles partially filling the voids between them. The resulting composite is then carbonized under a protective atmosphere and the carbon replica is obtained by dissolution of the $\mathrm{SiO}_{2}$ template in $\mathrm{HF}$ solution. Due to the spherical $\mathrm{SiO}_{2}$ particles constituting the template, the carbon replica exhibits spherical pores in the nanometer regime. An important feature is that pore connectivity is ensured because the silica particles are fused together.

As the particle size of the silica template can be controlled by a flame pyrolysis process, the pore size of the carbon replica can 


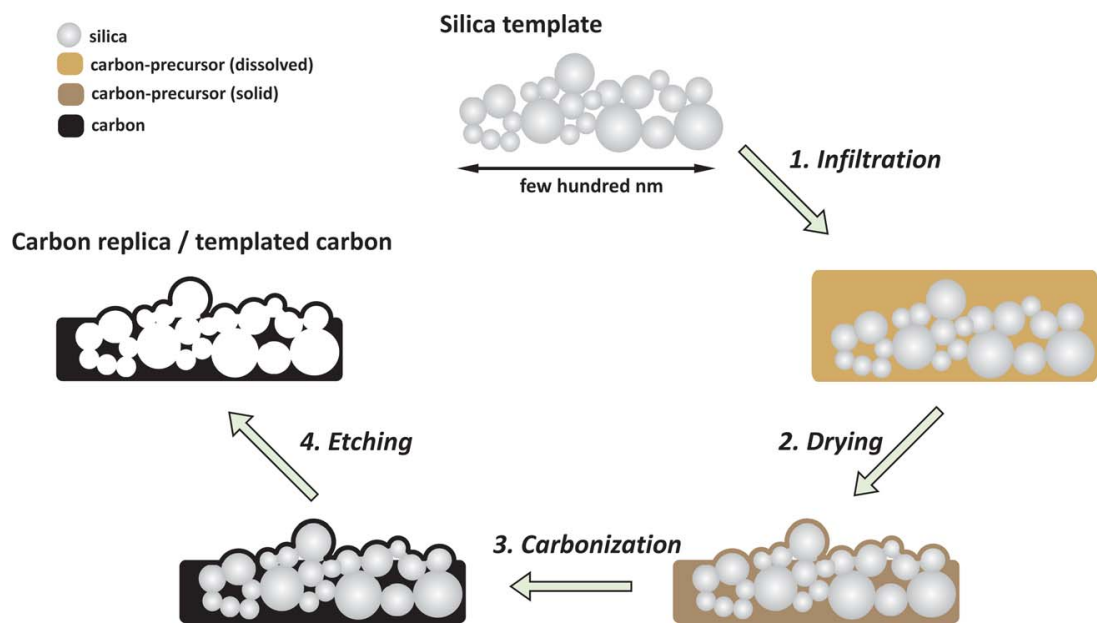

Fig. 1 Principle of the nanocasting process using fused spherical silica particles as a template.

be adjusted: a large average primary particle size correlates with a relatively high density of the silica template. The corresponding carbon replica then exhibits larger pores. Accordingly, a silica template consisting of small primary particles gives rise to a carbon replica with smaller pore sizes. To study the size effect, four silica templates (Template1-4) and corresponding carbon replicas (Carbon1-4) were prepared in this study. To illustrate the effect of the carbon precursor, one additional sample was prepared using sucrose as carbon source.

\subsection{Synthesis and structural characterization}

Fig. 2 shows typical SEM images of the template and the corresponding carbon replica. The silica particles are of perfect spherical shape and show a relatively broad size distribution ranging from around $50 \mathrm{~nm}$ to a few hundred $\mathrm{nm}$. The silica particles are randomly fused to form aggregates and mechanically stable, macroscopic structures. The voids between the particles are in the size regime of larger meso- $(2 \mathrm{~nm}<d<50 \mathrm{~nm})$ and macropores $(d>50 \mathrm{~nm})$. The carbon replica shows that the
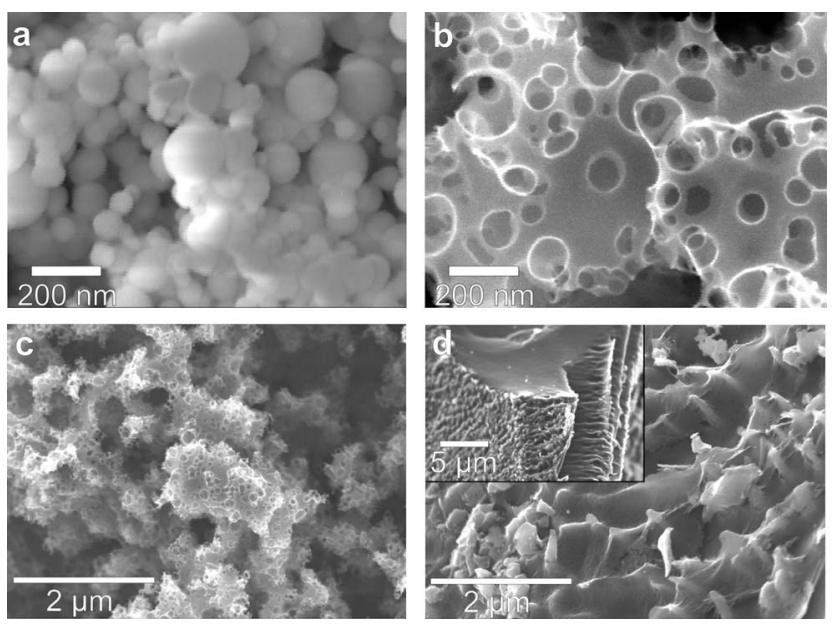

Fig. 2 SEM images of (a) silica template, (b and c) carbon replica based on mesophase pitch as carbon precursor, and (d) mesophase pitch carbonized without a template. morphology of the template is well replicated and a macroporous structure with interconnected, spherical pores in the larger mesoand macropore range is found. This structure is different from earlier works published in this field where powders of spherical silica nanoparticles were used to prepare predominantly mesoporous carbons $\mathrm{s}^{22-29}$ or carbons with larger macropores. ${ }^{22}$ Macroscopically, the prepared carbon materials consist of flakes in the $\mathrm{mm}$ range (Fig. S2†).

The composition and purity of the carbon materials were investigated by XPS (surface analysis) and by elemental analysis (bulk analysis). A XPS survey spectrum of the synthesized carbon material is shown in Fig. 3a. Carbon with the dominant peak at $285.0 \mathrm{eV}(\mathrm{C} 1 \mathrm{~s})$ and oxygen $(\mathrm{O} 1 \mathrm{~s}, 531.8 \mathrm{eV})$ were the only detectable species. The absence of signals corresponding to $\mathrm{Si}$ ( $\mathrm{Si} 2 \mathrm{p}$ at $99.8 \mathrm{eV}$ and $\mathrm{Si} 2 \mathrm{~s}$ at $150.2 \mathrm{eV}$ ) evidences that complete removal of the silica template was achieved. A carbon content of $95.4 \mathrm{at} \%(94.0 \mathrm{wt} \%)$ and an oxygen content of $4.6 \mathrm{at} \%(6.0 \mathrm{wt} \%)$ were derived from the $\mathrm{C} 1 \mathrm{~s}$ and $\mathrm{O} 1 \mathrm{~s}$ signals, corresponding to an atomic oxygen/carbon ratio of $\mathrm{O} / \mathrm{C}=0.048$. Bulk $\mathrm{C}, \mathrm{H}, \mathrm{N}, \mathrm{S}$ and $\mathrm{O}$ contents of the carbon samples as determined by elemental analysis were typically $>92 \mathrm{wt} \% \mathrm{C}, 1.4 \mathrm{wt} \% \mathrm{H}, 0.13 \mathrm{wt} \% \mathrm{~S}$ and $2-5 \mathrm{wt} \% \mathrm{O}$, corresponding to atomic ratios of $\mathrm{H} / \mathrm{C}=0.18$ and $\mathrm{O} / \mathrm{C}=0.02-0.04$, respectively. The nitrogen content was negligible.

As a reference, mesophase pitch was also carbonized without the presence of the silica template (non-templated carbon). Carbonization of bulk mesophase pitch gives rise to
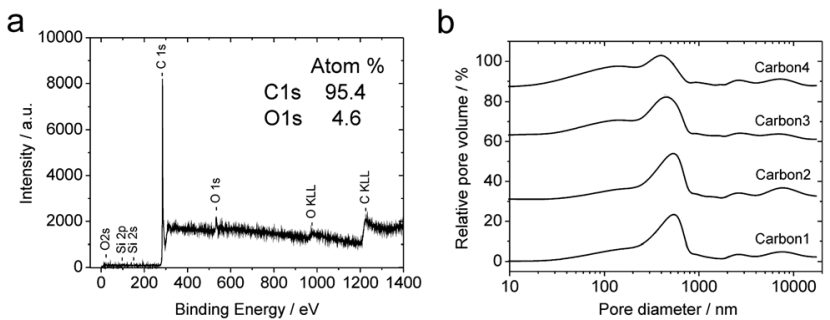

Fig. 3 (a) XPS survey spectrum of the carbon replica (Carbon1) and (b) pore size distribution of the different carbon replicas derived by $\mathrm{Hg}$ porosimetry. 
a macroscopic solid foam with pores of several millimeters in size (Fig. S3 $\dagger$ ). These very large pores are a result of the carbonization process that involves the release of various gaseous compounds upon heating while passing through a viscous state. ${ }^{30}$ For the present context this phenomenon is not relevant as all samples were ground for further use and porosity in this size regime is disrupted. Still, it is interesting to note that the foam formation was not observed when carbonizing pitch in the presence of the template, indicating that the carbonization process is influenced by the confinement in the silica matrix. The difference in porosity in the nano- and micrometer regime can be clearly seen from the SEM images in Fig. 2c and d. Carbonization of mesophase pitch without a template gives rise to a nonporous, low surface area carbon material, i.e. it can be concluded that the porosity observable in the SEM is due to the template and no intrinsic porosity in the nanometer regime develops during carbonization due to the carbon precursor. The elemental composition of the non-templated carbon as derived from XPS measurements was $99.1 \mathrm{at} \% \mathrm{C}$ and $0.9 \mathrm{at} \% \mathrm{O}$, i.e. an atomic ratio of $\mathrm{O} / \mathrm{C}=0.01$ is found. $\mathrm{O} / \mathrm{C}$ and $\mathrm{H} / \mathrm{C}$ ratios determined by elemental analysis were 0.02 and 0.18 , respectively. Comparing this with the results obtained for the templated carbons evidences that most of the functional groups containing oxygen stem from the nanocasting process.

Fig. $3 \mathrm{~b}$ shows the pore size distributions derived from $\mathrm{Hg}$ porosimetry of the four carbon replicas. Here it is important to note that, precisely speaking, mercury infiltration of pores is related to the pore orifice rather than the pore diameter. Nevertheless, $\mathrm{Hg}$ porosimetry gives a good estimate of the bulk sample porosity over a wide size regime, which is particularly important for samples containing larger pores (a few hundred $\mathrm{nm}$ ) not being detectable by nitrogen physisorption. The pore size distributions are broad for all samples, but exhibit defined maxima in the macropore range. In line with the concept shown in Fig. 1, the pore size can be adjusted by the silica template. For the present samples, this means that the pore size can be tailored in the range between $300 \mathrm{~nm}$ and $700 \mathrm{~nm}$. Total pore volumes of the carbon samples were typically between $2 \mathrm{~cm}^{3} \mathrm{~g}^{-1}$ and $3 \mathrm{~cm}^{3} \mathrm{~g}^{-1}$, as derived from $\mathrm{Hg}$ porosimetry.

While $\mathrm{Hg}$ porosimetry and SEM are not suitable for characterization of porosity in the micropore $(d<2 \mathrm{~nm})$ and small mesopore range, the samples were further investigated with $\mathrm{N}_{2}$ physisorption. Similar isotherms were found for the different templates and the corresponding carbon replica. As an example,
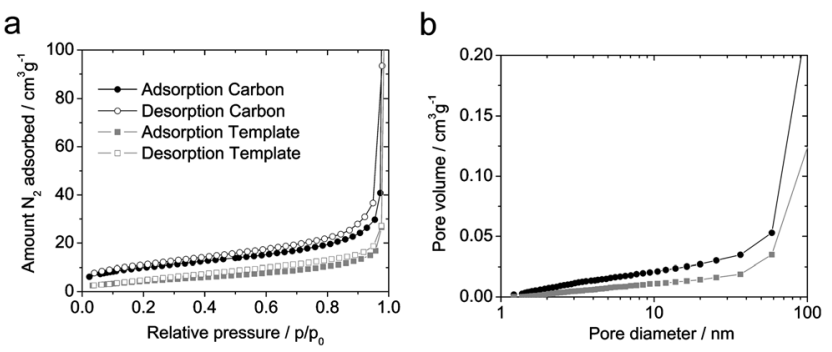

Fig. 4 Typical nitrogen physisorption isotherms (a) and cumulative pore volumes (b) for the silica template (Template1) and corresponding carbon replica (Carbon1). The cumulative pore volumes are derived from the adsorption branch of the isotherm (BJH model). the isotherms of silica template (Template1) and the corresponding carbon replica (Carbon1) are shown in Fig. 4. The isotherms show that the templates are predominantly macroporous as seen by the steep increase in $\mathrm{N}_{2}$ uptake at high relative pressures. BET specific surface areas of around $15-20 \mathrm{~m}^{2} \mathrm{~g}^{-1}$ were found for all silica samples indicating that the silica primary particles are practically non-porous and the porosity originates from their structural assembly only. Upon infiltration with the carbon precursor, the surface area further decreases to around 3-4 $\mathrm{m}^{2} \mathrm{~g}^{-1}$. The carbon replica shows similar isotherms, but the larger offset at small relative pressures is indicative of some microporosity. Typically surface areas between $35 \mathrm{~m}^{2} \mathrm{~g}^{-1}$ and $65 \mathrm{~m}^{2} \mathrm{~g}^{-1}$ were found for the carbon samples.

In contrast to the macropore diameter, where a direct correlation between the template and replica is observed, no correlation for the surface area is found. The carbon precursor itself barely contributes to the increased surface area values, as surface areas close to zero are typically found after carbonization without the silica template. Fig. 4 also shows the cumulative pore volumes derived from the adsorption branch of the isotherms using the $\mathrm{BJH}$ model. Clearly, the pore size distribution in the micropore and mesopore range is broad and the absolute volumes are comparatively small. A summary of the results obtained from the nitrogen physisorption measurements is given in Table 1.

Carbon replicas with higher surface area can be prepared by replacing mesophase pitch with sucrose as carbon source (CarbonSUC). A surface area of $S_{\mathrm{BET}}=530.8 \mathrm{~m}^{2} \mathrm{~g}^{-1}$ is found in this case, while preserving the same macroporous morphology. The increase in surface area can be seen from the large offset at low relative pressures, consequently the cumulative pore volumes are larger for pores in the nanometer regime (Fig. S4 $\dagger$ ). The larger surface area is related to the comparably poor carbonization behavior of sucrose, resulting in a defect rich carbon microstructure with large inherent microporosity. In contrast, mesophase pitch consists of polyaromatic moieties that carbonize well already at relatively low temperatures, thus minimizing intrinsic microporosity. The difference is clearly discernible from XRD shown in Fig. 5. Both carbon precursors exhibit a non-graphitic carbon microstructure with broad maxima around $2 \theta=26^{\circ}$ and $40-45^{\circ}$ corresponding to the interlayer and intralayer scattering of the graphene stacks, but the maxima are more distinct for the mesophase pitch based carbon. The more diffuse maxima in the XRD pattern of the sucrose based carbon indicate a low degree of structural order being correlated with the microporosity. A more detailed study on the carbon microstructure of mesophase pitch based carbon is given in ref. 13 .

In summary, silica materials prepared by flame pyrolysis allow the preparation of carbon materials with interconnected spherical macropores of tailored size. The surface area is sensitive to the carbon precursor used and can be significantly increased by replacing mesophase pitch by other carbon precursors such as sucrose.

An application where carbon materials with tailored porosity might be advantageous are batteries, for example when used as active anode material (enhanced kinetics for Li-insertion) or as support material for other active materials such as $\mathrm{LiFePO}_{4}{ }^{31-33}$ or sulfur. ${ }^{4,34}$ Here, we focus on the lithium storage properties of the prepared carbon materials and study the effect of the porosity in terms of rate capability and capacity. 
Table 1 Results from nitrogen physisorption. Total pore volumes were derived from the last point of the isotherm (which covers pores roughly smaller than $d<500 \mathrm{~nm}$ ). CarbonSUC is a carbon replica derived from sucrose as carbon precursor; all other carbon replicas were prepared using mesophase pitch as carbon precursor. Non-templated carbon is carbonized bulk mesophase pitch. The graphite is commercial SFG-44 (Timcal Ltd., Bodio, Switzerland)

\begin{tabular}{lcl}
\hline Sample & $\begin{array}{l}\text { BET surface area } \\
S_{\mathrm{BET}} / \mathrm{m}^{2} \mathrm{~g}^{-1}\end{array}$ & $\begin{array}{l}\text { Total pore } \\
\text { volume } / \mathrm{cm}^{3} \mathrm{~g}^{-1}\end{array}$ \\
\hline Template1 & 16.9 & 0.32 \\
Template2 & 15.1 & 0.30 \\
Template3 & 17.3 & 0.31 \\
Template4 & 17.4 & 0.21 \\
Carbon1 & 35.3 & 0.62 \\
Carbon2 & 67.7 & 0.60 \\
Carbon3 & 42.5 & 0.73 \\
Carbon4 & 33.9 & 0.69 \\
Graphite & 5.5 & 0.04 \\
Non-templated carbon & 0.4 & 0.002 \\
CarbonSUC & 530.8 & 2.14 \\
\hline
\end{tabular}

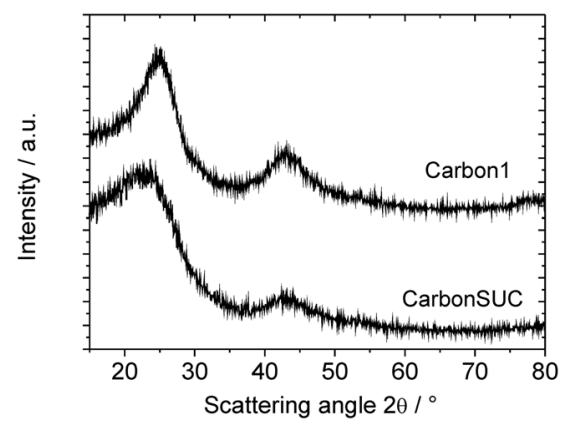

Fig. 5 XRD patterns of carbon derived from mesophase pitch (Carbon1) and sucrose (CarbonSUC).

\subsection{Electrochemical characterization}

Carbon electrodes were prepared by doctor blading a slurry of solvent (NMP), binder (PVDF) and carbon material onto a copper current collector. The carbon to binder weight ratio was $90: 10$. A SEM image of the electrode cross-section is shown in Fig. 6. The thickness of the porous carbon layer is approx. $40 \mu \mathrm{m}$. Galvanostatic lithium insertion $\left(\mathrm{CC}, Q_{\mathrm{cc}}\right)$ was followed by a potentiostatic step at $0.01 \mathrm{~V}(\mathrm{CV})$ to allow for relaxation of the system, giving the total capacity, $Q_{\text {total }}$. Lithium deinsertion was

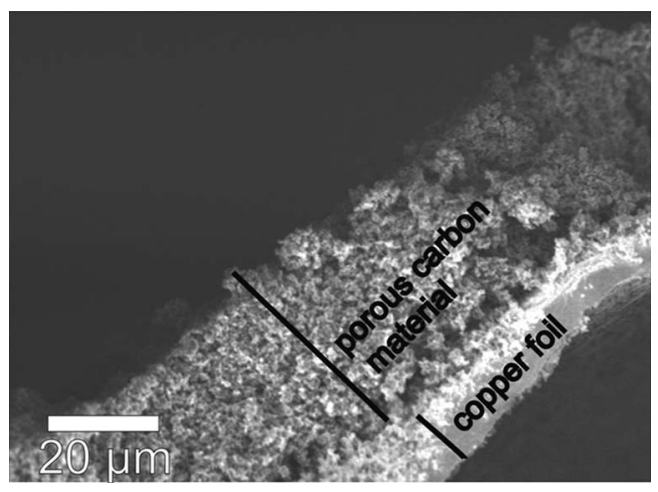

Fig. 6 SEM image of an electrode cross-section. done under galvanostatic conditions only. Fig. 7 shows results from the discharge (Li-insertion)/charge (Li-deinsertion) experiments conducted at a C-rate of C/5. Fig. 7a shows the voltage profile for sample Carbon2 and is representative for all samples investigated. The behavior is typical of non-graphitic carbon materials with SEI formation at potentials around 1.0-0.6 V during the first Li-insertion step, resulting in a large irreversible capacity. ${ }^{35-37}$ The first reversible capacity is in the order of $Q_{\text {total }}=700 \mathrm{~mA} \mathrm{~h} \mathrm{~g}^{-1}$, i.e. approximately twice the theoretical capacity of graphite $\left(372 \mathrm{~mA} \mathrm{~h} \mathrm{~g}^{-1}\right)$. Upon subsequent cycling the capacity stabilizes at around $550 \mathrm{~mA} \mathrm{~h} \mathrm{~g}^{-1}$. The large irreversible capacity is undesirable for practical applications but could be reduced by further pre-conditioning such as heat treatment under a selected atmosphere, for example. ${ }^{38,39}$

To view these results in the right perspective, we prepared electrodes from commercial graphite and from non-templated carbon under identical conditions. At a C-rate of $\mathrm{C} / 5$ the graphite reference showed stable capacity values around $310 \mathrm{~mA} \mathrm{~h} \mathrm{~g}$. The non-templated carbon exhibited the lowest capacities, after 10 cycles the capacity was only about one-fourth that of the templated carbons. Clearly, introducing porosity by the nanocasting process significantly increases the capacity of the carbon materials, even though no trend between the different macropore sizes and capacity was observed. Another possible
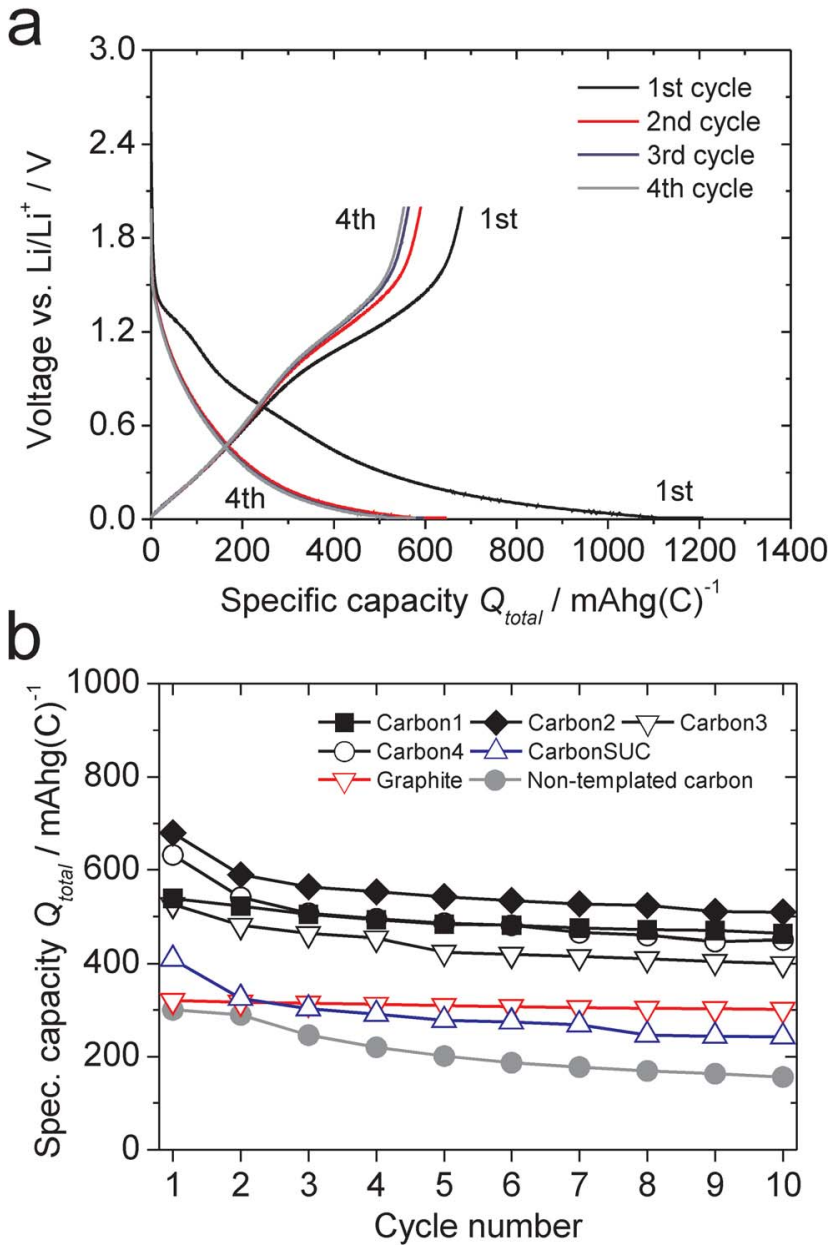

Fig. 7 Charge/discharge measurements at $\mathrm{C} / 5$, i.e. $i_{\mathrm{CC}}=74.4 \mathrm{mAg}(\mathrm{C})^{-1}$. (a) Voltage profile and (b) capacity (Li-insertion) vs. number of cycles. 

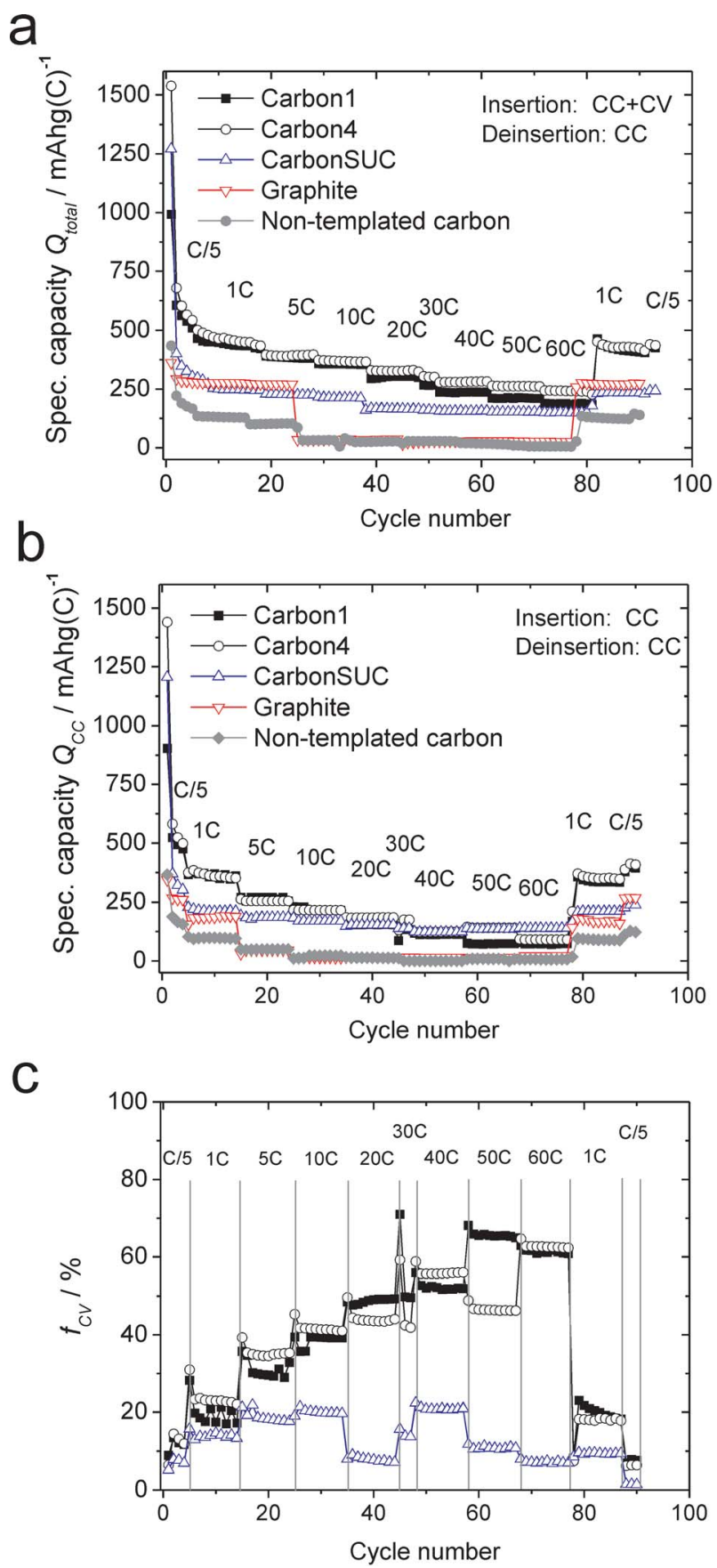

Fig. 8 (a) Rate capability measurements and cycling stability of the MP based porous carbons Carbon1 and Carbon4, the sucrose based porous carbon (CarbonSUC), carbonized MP (non-templated carbon) and graphite. (b) Capacities $Q_{\mathrm{cc}}$ under galvanostatic conditions. (c) Fraction $f_{\mathrm{CV}}$ in $\%$ of $Q_{\mathrm{total}}$ that is due to the potentiostatic step. $f_{\mathrm{CV}}=\left(Q_{\mathrm{CV}} / Q_{\text {total }}\right) \times 100 \%=\left(\left(Q_{\text {total }}-Q_{\mathrm{CC}}\right) / Q_{\text {total }}\right) \times 100 \%$.

explanation for the larger capacity might be the preferred orientation of the graphenes towards the surface. Hurt et al. showed that the discotic mesogenic structures constituting mesophase pitch show preferred "edge-on" orientation on many substrates, including quartz. ${ }^{40,41}$ Preservation of this orientation during carbonization would - after etching of the $\mathrm{SiO}_{2}$ - directly lead to a favorable orientation of the graphenes towards the surface and hence to facilitated lithium insertion/deinsertion. ${ }^{42,43}$ Preferred orientation of pitch on porous silica was also reported by Zhao et al. ${ }^{44}$
The advantage in using mesophase pitch as a carbon precursor also becomes clear when comparing the capacities with the sucrose based sample. Featuring a comparable interconnected macropore structure but larger surface area, the capacities are significantly lower compared to the pitch based sample. This observation indicates that the beneficial lithium insertion properties are due to both the interconnected porosity and the carbon precursor used.

The rate capabilities of mesophase pitch (MP) based samples with the most differing macropore sizes (Carbon1 and Carbon4), 
non-templated carbon, graphite and the sucrose based carbon are compared in Fig. 8. Total capacity $Q_{\mathrm{total}}$ and $Q_{\mathrm{CC}}$ are shown in Fig. 8a and b, respectively. Fig. 8c shows the fraction $f_{\mathrm{CV}}$ of the total capacity that is due to the potentiostatic step. Excellent rate capability and structural stability was found for both MP samples with capacities close to $Q_{\mathrm{CC}}=270 \mathrm{~mA} \mathrm{~h} \mathrm{~g}^{-1}\left(Q_{\text {total }}=\right.$ $\left.400 \mathrm{~mA} \mathrm{~h} \mathrm{~g}^{-1}\right)$ at a C-rate of $5 \mathrm{C}$ and around $Q_{\mathrm{CC}}=90 \mathrm{~mA} \mathrm{~h} \mathrm{~g}^{-1}$ $\left(Q_{\text {total }}=250 \mathrm{~mA} \mathrm{~h} \mathrm{~g}{ }^{-1}\right)$ at $60 \mathrm{C} . f_{\mathrm{CV}}$ steadily increases from around $10 \%$ at $\mathrm{C} / 5$ to $60 \%$ at $60 \mathrm{C}$. The non-porous references show a comparatively insufficient rate capability. Applying C-rates exceeding $5 \mathrm{C}$ leads to capacities between $Q_{\text {total }}=0 \mathrm{~mA}$ $\mathrm{h} \mathrm{g}^{-1}$ and $30 \mathrm{~mA} \mathrm{~h} \mathrm{~g}^{-1}$ only. Excellent cycling properties were also found for the sucrose based sample. Here the absolute capacities $Q_{\text {total }}$ are lower compared to the MP based samples, but the decrease upon increasing current is less severe. In fact, CarbonSUC exhibits higher galvanostatic capacities, $Q_{\mathrm{CC}}$, at C-Rates exceeding $30 \mathrm{C}$. Interestingly, $f_{\mathrm{CV}}$ is smaller compared to the MP based samples and does not directly correlate with the applied current (Fig. 8c), this indicates even more rapid kinetics.

Ideally, one would like to directly correlate the rate performance of the various samples with their porosity and microstructure. So far it can be concluded from the present results that the high capacity and excellent rate capability of the MP based carbons are due to the carbon precursor and the porosity introduced by the nanocasting process. The differences in pore size are, however, so far not large enough to find significant differences in the electrochemical performance. Though the hard carbon CarbonSUC with higher surface area shows lower total capacities $Q_{\mathrm{total}}$, its rate performance is still excellent and $f_{\mathrm{CV}}$ is comparatively small, i.e. this carbon provides in total less, but more rapidly accessible Li storage sites compared to the MP based carbons. Whether this behavior is an effect of the higher surface area (capacitive effects) or also related to other factors such as carbon microstructure, binder content, average diffusion distance within the carbon microstructure, etc. still needs to be clarified. On the other hand, one can draw conclusions by comparing the present results with our earlier findings on another mesophase pitch based, templated carbon material with an interconnected macroporous structure. ${ }^{14}$ In that case, the material additionally exhibited a larger amount of small, well defined mesopores $\left(V_{\text {meso }}(d \leq 10 \mathrm{~nm})=0.5 \mathrm{~cm}^{3} \mathrm{~g}^{-1}\right.$ compared to approx. $0.025 \mathrm{~cm}^{3} \mathrm{~g}^{-1}$ in this work) and consequently also a higher surface area $\left(S_{\mathrm{BET}}=330 \mathrm{~m}^{2} \mathrm{~g}^{-1}\right.$ compared to approx. $35-70 \mathrm{~m}^{2} \mathrm{~g}^{-1}$ in this work). As the capacities $Q_{\mathrm{CC}}$ and rate capabilities are comparable, it becomes evident that the excellent performance is determined to a lesser extent by the mesoporosity, but is probably rather a result of the interconnected macroporous $(d>50 \mathrm{~nm})$ network prepared by the nanocasting process. Obviously, further studies on the pore size effect are needed to clarify the effect of porosity and carbon microstructure on the rate performance.

\section{Conclusion}

The nanocasting process was used to prepare porous carbon materials with interconnected, spherical macropores based on industrial, monolithic silica templates. The structure resembles the Porocarb ${ }^{\circledR}$ carbon material that has been recently registered as a trademark and is reproducibly prepared on the kilogram scale. While the average pore size can be tailored by the silica template, surface area and microporosity can be easily increased by the choice of appropriate carbon precursors. In this way high surface area carbons can be prepared using sucrose as carbon precursor while carbons with lower surface area and more welldefined carbon microstructure are obtained when mesophase pitch is used as carbon source. Furthermore, our study proves that hard-templating is indeed a feasible industrial process for the generation of porous non-graphitic carbon materials, which allays concerns that the usage of HF or strong bases is a major obstacle to this route.

The beneficial effect of porosity on charge storage properties was illustrated by lithium insertion experiments. When used as an anode material in half cells, the templated carbons show rapid kinetics compared to the non-porous references, illustrating the beneficial effect of porosity on the transport properties. Interestingly, the excellent rate performance of the MP based samples prepared by nanocasting seems determined by the macroporosity rather than high surface area and/or large micro- and mesopore content.

\section{Acknowledgements}

We thank Jürgen Janek for scientific discussion on the manuscript and C. Raiss, K. Peppler, L. Chuenchom, H. Metelmann and $\mathrm{C}$. Weidmann for assistance with the elemental analysis, SEM, $\mathrm{Hg}$ porosimetry, XPS and $\mathrm{N}_{2}$ physisorption measurements.

\section{References}

1 R. Ryoo, S. H. Joo and S. Jun, J. Phys. Chem. B, 1999, 103, 77437746.

2 J. Lee, S. Yoon, T. Hyeon, S. M. Oh and K. B. Kim, Chem. Commun., 1999, 2177-2178.

3 P. Adelhelm and P. E. de Jongh, J. Mater. Chem., 2011, 21, 24172427.

4 X. Ji, K. T. Lee and L. F. Nazar, Nat. Mater., 2009, 8, 500-506.

5 A. Taguchi and F. Schuth, Microporous Mesoporous Mater., 2005, 77, $1-45$.

6 K. Xia, Q. Gao, C. Wu, S. Song and M. Ruan, Carbon, 2007, 45, 1989-1996.

7 H. S. Zhou, S. M. Zhu, M. Hibino, I. Honma and M. Ichihara, $A d v$. Mater., 2003, 15, 2107.

8 R. Ryoo, S. H. Joo, M. Kruk and M. Jaroniec, Adv. Mater., 2001, 13, 677-681.

9 B. Sakintuna and Y. Yurum, Ind. Eng. Chem. Res., 2005, 44, $2893-$ 2902.

10 Y. D. Xia, Z. X. Yang and R. Mokaya, Nanoscale, 2010, 2, 639-659.

11 J. Biener, M. Stadermann, M. Suss, M. A. Worsley, M. M. Biener, K. A. Rose and T. F. Baumann, Energy Environ. Sci., 2011, 4, 656667.

12 A. H. Lu, D. Zhao and Y. Wan, Nanocasting: A Versatile Strategy for Creating Nanostructured Porous Materials, Royal Soc of Chemistry, Cambridge, 2009.

13 P. Adelhelm, K. Cabrera and B. M. Smarsly, Sci. Technol. Adv. Mater., 2012, 13, 015010.

14 Y. S. Hu, P. Adelhelm, B. M. Smarsly, S. Hore, M. Antonietti and J. Maier, Adv. Funct. Mater., 2007, 17, 1873-1878.

15 S. Wenzel, T. Hara, J. Janek and P. Adelhelm, Energy Environ. Sci., 2011, 4, 3342-3345.

16 P. Adelhelm, Y. S. Hu, L. Chuenchom, M. Antonietti, B. M. Smarsly and J. Maier, Adv. Mater., 2007, 19, 4012.

17 K. Tang, R. J. White, X. Mu, M.-M. Titirici, P. A. van Aken and J. Maier, ChemSusChem, 2012, 5, 400-403.

18 C. Neumann, WO/2011/0912149, 2011. 
19 B. Hannebauer and F. Menzel, Z. Anorg. Allg. Chem., 2003, 629, $1485-1490$

20 S. E. Pratsinis, Prog. Energy Combust. Sci., 1998, 24, 197-219.

21 G. D. Ulrich, Combust. Sci. Technol., 1971, 4, 47.

22 M. M. Titirici, A. Thomas and M. Antonietti, Adv. Funct. Mater. 2007, 17, 1010-1018.

23 S. J. Han and T. Hyeon, Chem. Commun., 1999, 1955-1956.

24 Z. Li and M. Jaroniec, Carbon, 2001, 39, 2080-2082.

25 Z. Li and M. Jaroniec, J. Am. Chem. Soc., 2001, 123, 9208-9209.

26 Z. Li and M. Jaroniec, Chem. Mater., 2003, 15, 1327-1333.

27 J. Eric Hampsey, Q. Hu, L. Rice, J. Pang, Z. Wu and Y. Lu, Chem. Commun., 2005, 3606-3608.

28 W. Li, D. Chen, Z. Li, Y. Shi, Y. Wan, G. Wang, Z. Jiang and D. Zhao, Carbon, 2007, 45, 1757-1763.

29 Z. Lei, Y. Xiao, L. Dang, S. Bai and L. An, Microporous Mesoporous Mater., 2008, 109, 109-117.

30 S. Z. Li, Y. M. Tian, Y. J. Zhong, X. Yan, Y. Song, Q. G. Guo, J. L. Shi and L. Liu, Carbon, 2011, 49, 618-624.

31 C. M. Doherty, R. A. Caruso, B. M. Smarsly, P. Adelhelm and C. J. Drummond, Chem. Mater., 2009, 21, 5300-5306.

32 G. Wang, H. Liu, J. Liu, S. Qiao, G. M. Lu, P. Munroe and H. Ahn, Adv. Mater., 2010, 22, 4944.

33 M. Yang and Q. Gao, J. Alloys Compd., 2011, 509, 3690-3698.
34 N. Jayaprakash, J. Shen, S. S. Moganty, A. Corona and L. A. Archer, Angew. Chem., Int. Ed., 2011, 50, 5904-5908.

35 J. R. Dahn, T. Zheng, Y. H. Liu and J. S. Xue, Science, 1995, 270, 590-593.

36 Z. Ogumi and H. Wang, in Lithium-Ion Batteries, ed. M. Yoshio, R. J. Brodd and A. Kozawa, Springer, 2009, p. 49.

37 M. Winter, J. O. Besenhard, M. E. Spahr and P. Novak, Adv. Mater., 1998, 10, 725-763.

38 W. M. Lu and D. D. L. Chung, Carbon, 2003, 41, 945-950.

39 E. Buiel and J. R. Dahn, Electrochim. Acta, 1999, 45, 121-130.

40 K. Jian, T. C. Truong, W. P. Hoffman and R. H. Hurt, Microporous Mesoporous Mater., 2008, 108, 143-151.

41 K. Q. Jian, H. S. Shim, D. Tuhus-Dubrow, S. Bernstein, C. Woodward, M. Pfeffer, D. Steingart, T. Gournay, S. Sachsmann, G. P. Crawford and R. H. Hurt, Carbon, 2003, 41, 2073-2083.

42 H. D. Abruna, Y. Kiya and J. C. Henderson, Phys. Today, 2008, 61, 43- 47.

43 F. Guo, A. Mukhopadhyay, B. W. Sheldon and R. H. Hurt, Adv. Mater., 2011, 23, 508.

44 H. F. Yang, Y. Yan, Y. Liu, F. Q. Zhang, R. Y. Zhang, Y. Meng, M. Li, S. H. Xie, B. Tu and D. Y. Zhao, J. Phys. Chem. B, 2004, 108, 17320-17328. 